\title{
REVIEW ARTICLE OPEN Engineering 3D approaches to model the dynamic microenvironments of cancer bone metastasis
}

\author{
Han Qiao ${ }^{1}$ and Tingting Tang ${ }^{1}$
}

\begin{abstract}
Cancer metastasis to bone is a three-dimensional (3D), multistep, dynamic process that requires the sequential involvement of three microenvironments, namely, the primary tumour microenvironment, the circulation microenvironment and the bone microenvironment. Engineered 3D approaches allow for a vivid recapitulation of in vivo cancerous microenvironments in vitro, in which the biological behaviours of cancer cells can be assessed under different metastatic conditions. Therefore, modelling bone metastasis microenvironments with 3D cultures is imperative for advancing cancer research and anti-cancer treatment strategies. In this review, multicellular tumour spheroids and bioreactors, tissue engineering constructs and scaffolds, microfluidic systems and $3 \mathrm{D}$ bioprinting technology are discussed to explore the progression of the 3D engineering approaches used to model the three microenvironments of bone metastasis. We aim to provide new insights into cancer biology and advance the translation of new therapies for bone metastasis.
\end{abstract}

Bone Research (2018)6:3; https://doi.org/10.1038/s41413-018-0008-9

\section{INTRODUCTION}

Bone metastasis is the major complication of advanced osteotropic cancers, including breast cancer (BC), prostate cancer (PC), lung cancer ( $\mathrm{LC}$ ) and multiple myeloma (MM). These metastases can cause significant morbidity due to skeletal-related events including pathological fracture, spinal cord compression, bone pain and hypercalcemia. In addition, metastatic bone lesions contribute to a poor prognosis, despite current therapeutic strategies. ${ }^{1-3}$ Hence, it is imperative to develop novel effective treatments for bone metastasis through a better understanding of malignant bone metastases in the clinical setting. Cancer cells naturally inhabit a three-dimensional (3D) architecture within host microenvironments. Currently, two-dimensional (2D) culture biosystems fail to consider the dynamic interactions between cancer cells and the microenvironment, and these systems differ from actual 3D biostates in regulating the genotypic and phenotypic bioactivity of malignant cells. Studies involving 3D biosystems over the past several decades have significantly bridged the gap between 2D culturing patterns and in vivo animal models. ${ }^{4-6}$ Hence, it is important to take advantage of spatial approaches in bone metastasis research to emphasise the dynamic dialogue between cell-cell and cell-extracellular matrix (ECM) interactions.

To date, the evolution of malignant bone metastasis has classically been characterised as a dynamic multistep process, namely, the invasion-metastasis cascade, in which cancer cells undergo a sequential journey of primary tumour transformation, local invasion, intravasation, survival in circulation, extravasation and metastatic colonisation in a distant bone microenvironment. ${ }^{7}$ Stephen Paget proposed the "seed and soil" hypothesis, ${ }^{8}$ which suggests that cancer cell metastasis is akin to the dissemination of plant seeds. To better understand the underlying biology of bone metastasis, separation of the complex cascade into several more explicit and foreseeable systems is required. Herein, we expand the connotation of "soil" to a wider range consisting of the following three microenvironments during cancer bone metastasis: the primary tumour microenvironment (PTM), circulation microenvironment (CM) and bone microenvironment (BM) (Fig. 1). Establishing the most representative $3 \mathrm{D}$ microenvironment is imperative and requires a comprehensive understanding of the application of 3D approaches in cancer research.

\section{OVERVIEW OF BONE METASTATIC MICROENVIRONMENTS}

In the PTM, the advent of a compatible metastasis frequently occurs in osteotropic tumour cells with limitless proliferative capability in primary sites; in this process, angiogenesis is critically important. ${ }^{9}$ When there are metabolic stresses on the tumour cells, the equilibrium between pro- and anti-angiogenic factors is altered, leading to recruitment of endothelial cells and fibroblasts, which form new vessels from the surrounding stroma. ${ }^{10}$ Angiogenesis not only satisfies the heightened metabolic needs of cancer cells but also supports avenues for local infiltration and foreign dissemination. Furthermore, another critical event in promoting cancer cell metastasis is epithelial-mesenchymal transition (EMT). In response to various extracellular EMTinducing signals, potential metastatic cells orchestrate invasionpromoting molecular, cellular and morphological changes through a cellular transformation from an epithelial phenotype with apical-basal polarisation to a mesenchymal phenotype with high motility capability and a spindle shape. ${ }^{11}$ Then, the cells access vascular systems with the assistance of newly formed microcapillaries, resulting in the onset of subsequent cellular events in the CM.

\footnotetext{
${ }^{1}$ Shanghai Key Laboratory of Orthopaedic Implants, Department of Orthopaedic Surgery, Shanghai Ninth People's Hospital, Shanghai Jiao Tong University School of Medicine, Shanghai 200011, China

Correspondence: Tingting Tang (ttt@sjtu.edu.cn)
}

Received: 27 April 2017 Revised: 1 December 2017 Accepted: 27 December 2017

Published online: 26 February 2018 

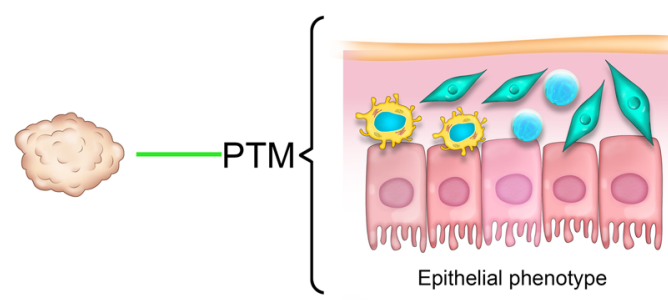

Epithelial phenotype
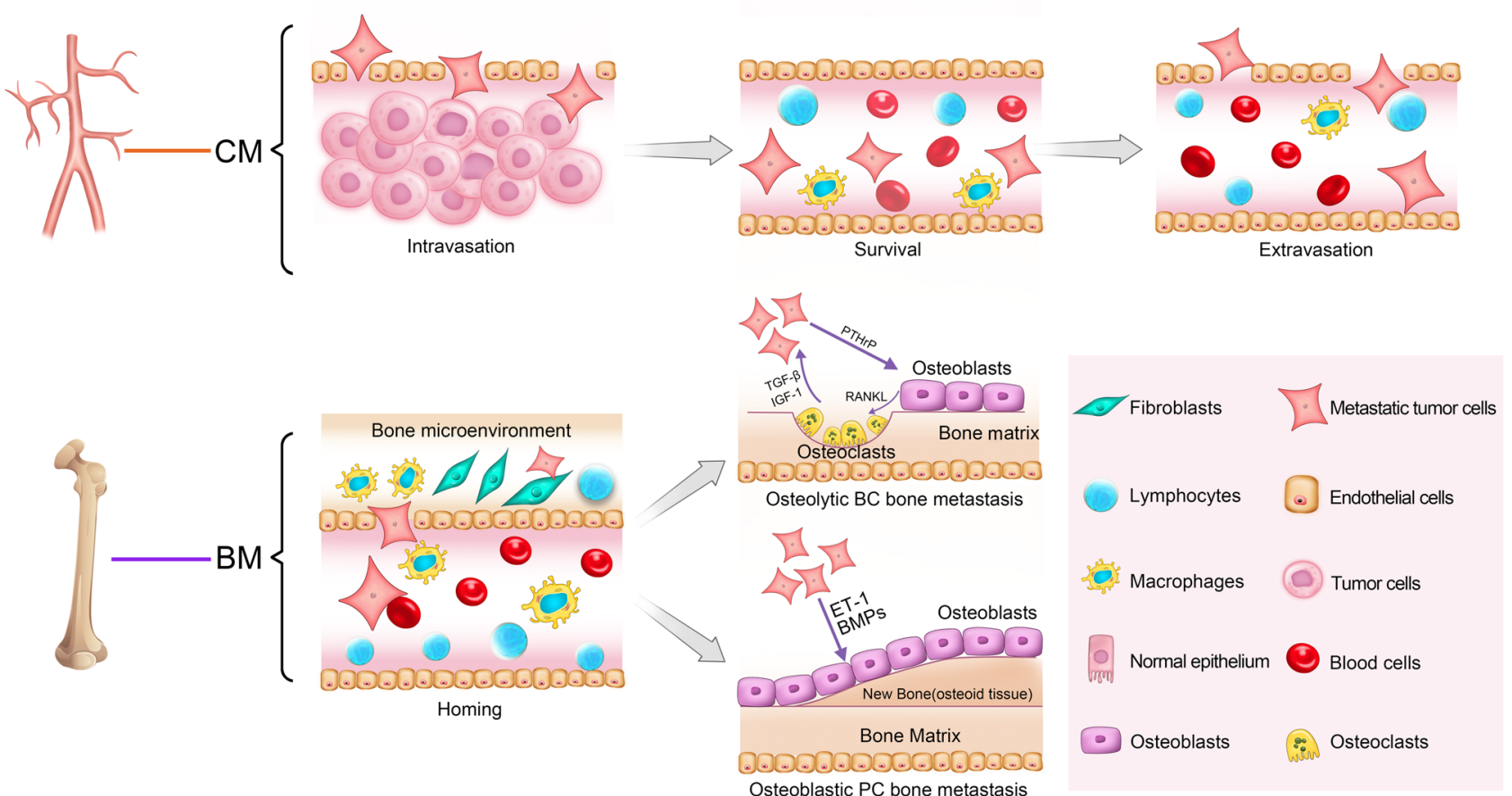

Fig. 1 Illustrations of three metastatic microenvironments during osteolytic and osteoblastic cancer bone metastases

The activity of cancer cells in the CM begins with intravasation and ends with extravasation. Intravasation involves a series of dynamic interactions between malignant cells and the microenvironment, such as decreased intercellular adhesion, increased cytoskeletal motility, active ECM remodelling and widened endothelial gaps, which accelerate the migratory pace of cancer cells to distant bone. Tumour cells that breach the normal vascular endothelium become circulating tumour cells (CTCS). With the substantial cell attrition induced by trans-membrane migration, fewer than $1 \%$ of original CTCS are capable of successful colonization in secondary sites. ${ }^{12}$ A number of immune cells, particularly macrophages and T-lymphocytes, obliterate CTCs within the CM. ${ }^{13}$ One example of how CTCs escape cell death in the CM is by inhibiting anoikis. Normally, the loss of cell-cell and cell-ECM interactions in migratory cells leads to cell apoptosis, but CTCs can overexpress a series of surface receptors to activate prosurvival pathways, ${ }^{14}$ resulting in their ability to survive in the CM through anoikis resistance.

The BM of bone tissues provides fertile "soil" for circulating osteotropic cancer cells to settle and populate. Metastatic bone lesions can generally be classified into osteolytic (such as in BC bone metastases) and osteoblastic (such as in PC bone metastases) microenvironments, which have distinct radiographic appearances. Osteoclasts are multinucleated cells derived from precursor osteoclasts of the monocyte-macrophage lineage. These cells dissolve calcium phosphate crystals and degrade ECM to demineralise bone structure by releasing relevant functional proteases. Osteoblasts are specialised, well-differentiated mononucleated cells derived from mesenchymal stem cells (MSCs) in the bone marrow cavity. These cells produce calcium phosphate crystals and generate newly formed ECM into matrix interstices for bone remineralization. ${ }^{15}$ Therefore, bone homoeostasis is maintained by the equilibrium between osteoblasts and osteoclasts, while its disruption may convert the BM from normal physiological niches to pathological metastatic niches. CTCs that enter the BM become disseminated tumour cells (DTCs), which can persist in dormancy for several years. ${ }^{16}$ Under conditions of increased stress/load, decreased immunity and stimulation by molecular signals, dormant DTCs progress to macrometastases, confirming the clinical significance of eliminating dormant cells in the BM to achieve long-term remission and overcome oncotherapy resistance.

Although osteocytes are the most abundant bone cells in bone tissues compared with osteoclasts and osteoblasts, little is known about their roles in osteotropic cancer bone metastasis. ${ }^{17}$ Osteocytes participate in osteoclastogenesis and osteoblastogenesis by secreting receptor activator of nuclear kappa B ligand (RANKL), osteoprotegerin (OPG), macrophage colony stimulating factor (M-CSF) and sclerostin. ${ }^{18}$ Therefore, targeting osteocytes to inhibit osteolytic RANKL expression abrogates early BC bone metastasis. ${ }^{19}$ In addition, serving as mechanical sensor cells, osteocytes react to shear stress and pressure from bone metastases, contributing to reciprocal tumour growth and osteoclast formation. . $^{21}$

For BC bone metastasis, once circulating cancer cells manage to survive in distant osseous tissues, cellular and extracellular components adapt to create a compatible niche by initiating a reciprocal vicious cycle between malignant cells and the bone microenvironment. This cycle in the BM involves mutual crosstalk between cancer cells and the microenvironment, and represents 


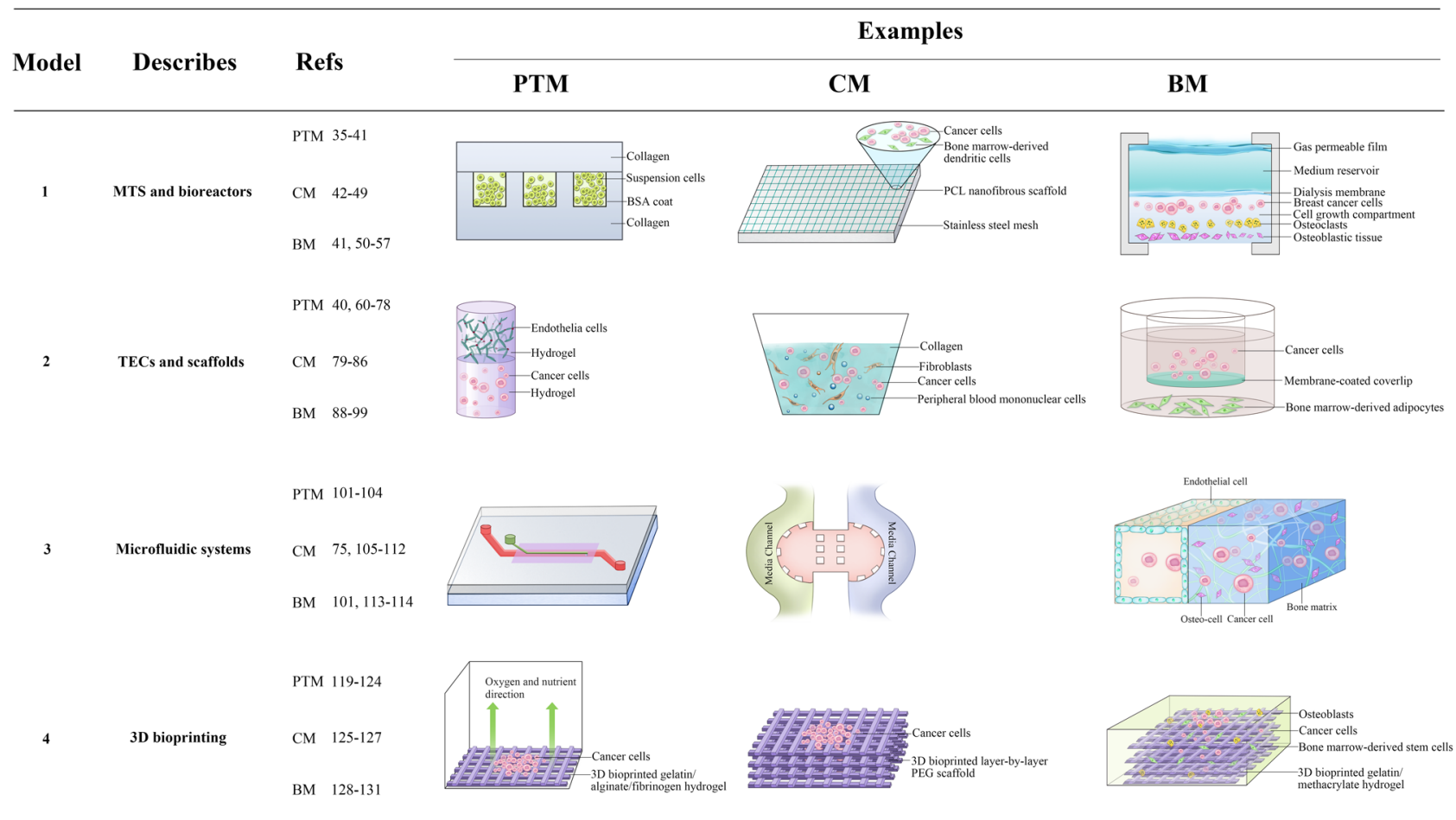

Fig. 2 Examples of in vitro engineering 3D approaches in modelling the dynamic microenvironments of cancer bone metastasis referenced in this review

the core implication of 3D approaches in cancer bone metastasis research. With respect to PC bone metastasis, tumour cells generate several growth factors, such as endothelin 1 (ET-1) and bone morphogenetic proteins (BMPs), that stimulate osteoblastic progenitor cell recruitment and maturation to produce new pathological bone, ${ }^{22}$ namely, immature mineralised bone (osteoid) around metastasis sites.

Collectively, numerous key players involved in bone metastasis, including biotic cellular components, such as tumour cells, endothelial cells, immune cells, osteoblasts, osteoclasts and osteocytes, and abiotic factors, such as growth factors and mechanical support, have been implicated in making metastatic microenvironments permissive for malignant cells. ${ }^{23}$ Cancer cells within metastatic bone microenvironments undergo a series of genetic and morphologic transformations that are stimulated by the extracellular microenvironment. ${ }^{24}$ However, with respect to 3D approaches, modelling the entire metastasis process within individual biosystems is difficult because metastatic microenvironments have varying yet specific crosstalk between cells and the surrounding milieu. ${ }^{25}$ Therefore, there are specific considerations for 3D approaches based on the processes of metastasis, ${ }^{26}$ making this review timely and useful.

\section{PARADIGM SHIFT: FROM 2D TO 3D}

Understanding the underlying cellular events during bone metastasis is a mandatory requirement for development of appropriate and efficacious metastatic cancer models. Following this paradigm, studies of bone metastasis traditionally utilised 2D cultures to tackle the maldistribution of nutrition that leads to proliferation inhibition of the studied cells. Cells in 2D cultures are introduced to excessive nutrients and oxygen through an increased exchange area, which compels these cells to immortalise instead of suffering programmed apoptosis after multiple in vitro passages. Further, cells that adhere to flat and rigid 2D substrates experience loss of cell polarisation, along with a modified amount, configuration and composition of ECM proteins.
This is of vital significance; the ECM is a crucial component of metastatic cancer microenvironments because the ECM exerts a functional influence on cancer cell proliferation, migration and apoptosis by modulating a series of soluble cytokines and signalling pathways. ${ }^{27}$ Additionally, the ECM communicates with cellular components frequently in vivo, while the lack of ECM results in diminished crosstalk between metastatic cells and the surrounding microenvironment that can lead to the disruptive establishment of bone metastasis. Moreover, monolayer cell cultures misrepresent whole-solid tumours for therapeutic drug development because 2D-cultured cells are normally immortalised and particularly vulnerable to drugs targeting rapidly dividing cells. In contrast, the tumour mass in vivo comprises a wide range of cellular states, including rapidly growing, stagnated, necrotic and apoptotic cells, which is a significant divergence from 2D cancer bone metastasis models.

To overcome these experimental constraints, researchers have developed multiple viable 3D approaches to model bone metastasis while maintaining reproducibility and enhancing complexity. These models have the following merits, which 2D approaches lack: (a) non-toxic, good biocompatibility that will not trigger subsequent inflammation or an immune response; (b) appropriate biodegradation rates that suit tissue regeneration rates; (c) easy processing while retaining hardness and mechanical strength to a certain degree, with a stable structural appearance; (d) adequate porosity and specific surface area; and (e) desirable surface activity to maintain viable cancer cell morphologies and phenotypes. $^{28-31}$ Unlike 2D cultures, 3D biosystems have a consistent spatial framework, which helps to directly correlate structures with functions. In addition, in 3D models, the components of the supporting matrix (such as a collagen scaffold and hybrid substrate) can simulate the loose or dense connective tissues surrounding host cells, thus creating a desirable milieu for studying the migration and invasion of metastatic cancer cells. The cellular phenotypes and signal transduction in 3D platforms are more similar to in vivo conditions than are those in $2 \mathrm{D}$ cultures, providing a reliable re-establishment of vivid crosstalk 
A

\section{Tissue maturation}

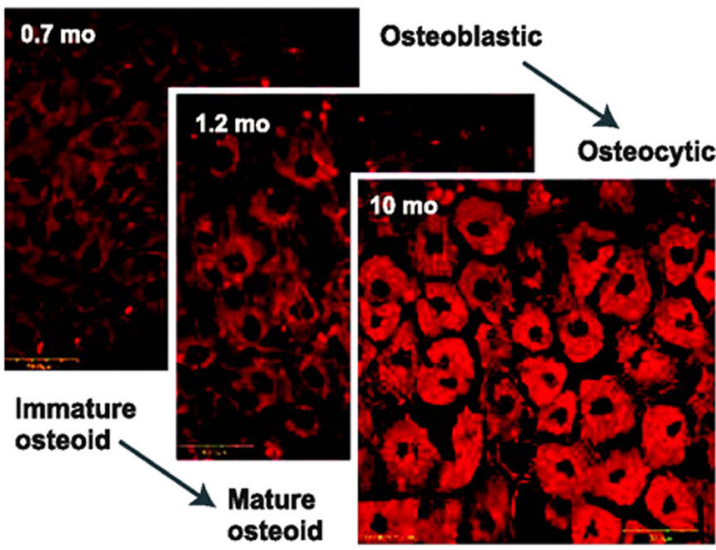

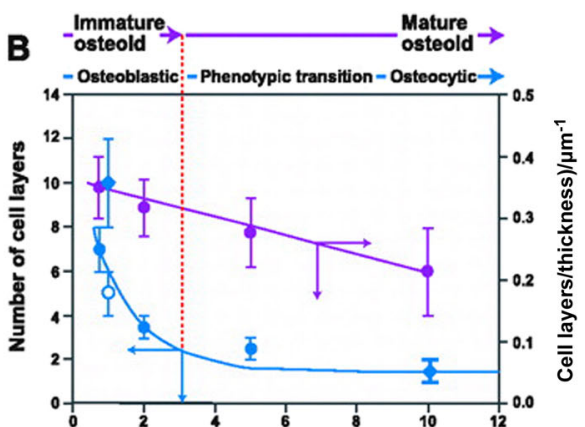

Months of MC3T3-E1 osteoid bioreactor culture

\begin{tabular}{|c|c|c|c|c|}
\multicolumn{4}{|c|}{ Months of MC3T3-E1 osteoid bioreactor culture } \\
\hline $\begin{array}{c}\text { Exporimental } \\
\text { parameter } \\
\text { Q BC:OB=1:10 }\end{array}$ & \multicolumn{4}{|c|}{ Months of bioreactor culture } \\
\cline { 2 - 5 } $\begin{array}{c}\text { BC } \\
\text { colonlzatlon }\end{array}$ & $(+++)$ & 1 & 2 & 5 \\
\hline $\begin{array}{c}\text { Tissue } \\
\text { ponotration }\end{array}$ & $(-)$ & $(+)$ & $(+)$ & $(+)$ \\
\hline BC filing & $(-)$ & $(+)$ & $(+)$ & $(+)$ \\
\hline $\begin{array}{c}\text { Tumor } \\
\text { formatlon }\end{array}$ & $(-)$ & $(+)$ & $(+)$ & $(+)$ \\
\hline
\end{tabular}

\section{Cancer colonization}
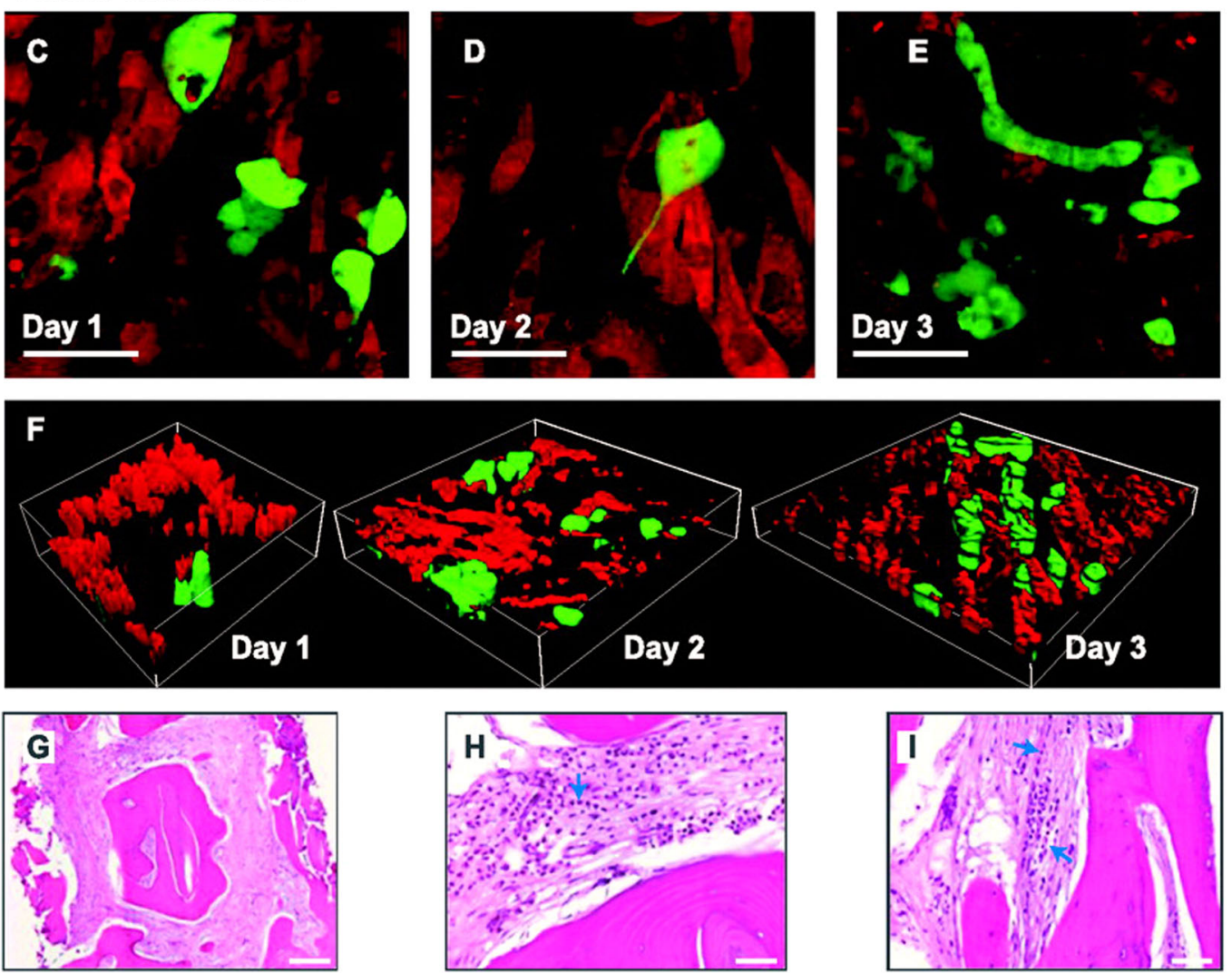

Fig. 3 a Osteogenic tissue maturation in the bioreactor recapitulates development of native bone by systematic and reproducible phenotypic maturation of preosteoblasts through mineralising osteoblasts to terminally differentiated osteocytes. b MC3T3-E1 cells produce and mineralise a thick, engulfing ECM that slowly decreases in thickness and number of cell layers through progressive apoptosis to a final stable state exhibiting no sign of tissue necrosis over 10 months of continuous culture (graph). c-f Interaction of MDA-MB-231 ${ }^{\mathrm{GFP}}$ human cancer cells (green, GFP) with osteogenic tissue (red, osteoblasts; black, ECM) depends on tissue maturity (b, table) and exhibits stages of cancer cell adhesion (c), penetration (d) and alignment of cancer cell into files (e) that are reminiscent of events observed in pathologic tissue. $\mathbf{f}$ Filing is especially evident in corresponding 3D confocal reconstructions. g-i For comparison, Indian Filing is shown in a section from bone (solid pink) with metastatic breast cancer (rows of cells with dark purple nuclei). The scale bars in a, c and d represent $50 \mu \mathrm{m}$, the scale bar in $\mathbf{f}$ represents $100 \mu \mathrm{m}$, the scale bar in $\mathbf{g}$ represents $200 \mu \mathrm{m}$, and the scale bars in $\mathbf{h}$ and $\mathbf{i}$ represent $50 \mu \mathrm{m}$. Reprinted with permission from ref. ${ }^{55}$ Copyright (2009) American Association for Cancer Research (AACR) Publishing Group 
between metastatic cancer cells and neighbouring matrices. ${ }^{32}$ Moreover, 3D systems promote rich cell-cell and cell-ECM interactions, which helps in the precise recapitulation of cancer bone metastasis at the tissue, cellular and molecular levels. In addition, multiple studies have illustrated that 3D approaches lead to a comprehensive understanding of the chemosensitivity of cells to therapeutic agents since these models represent actual solid tumour structures that consist of an inner necrotic core and an outer shell of proliferative metastatic cancer cells, contributing to a concentration gradient that is lowered from outside to inside. This gradient often hampers treatment effects, and a representative gradient model would be instrumental in developing clinically effective drugs. However, despite the aforementioned advantages of $3 \mathrm{D}$ models for studying bone metastasis, the ability of each individual biosystem to simulate a specific host microenvironment is quite different. For instance, collagen scaffolds lack dense tissues, and cell-derived scaffolds have larger porosity and are thinner than tissue-derived scaffolds, which enable cancer cells to metastasise relatively quickly compared with the host in vivo microenvironment. Therefore, these conditions require researchers to devise corresponding customised experimental toolkits for distinctive research purposes and to develop novel strategies to meet the demands of evolving studies.

\section{THREE-DIMENSIONAL APPROACHES TO MODEL CANCER BONE METASTASIS}

As mentioned above, it is important to re-establish the 3D cellular architecture and surrounding microenvironment for in vivo-like bone metastasis models. Based on the characterisation of the unique crosstalk between osteotropic cancer cells and the exterior microenvironment as well as diverse methods for 3D scaffold fabrication, we summarised several inspiring types of 3D approaches for recapitulating key cellular events within the three cancer microenvironments (PTM, CM and BM). Figure 2 shows these formats and offers selected references.

Multicellular tumour spheroids (MTSs) and bioreactors

Mixed cell MTS systems have become a promising approach since their development in the 1970s. MTSs can form cell aggregates with necrosis inside and an uneven distribution of growth cytokines and metabolic needs, resembling the natural characteristics of metastatic solid tumours. ${ }^{33,34}$ Furthermore, the combination of MTSs with relevant bioreactors recapitulates specialised and controllable metastatic microenvironments that consider the sophisticated crosstalk within and between cells.

Modelling cellular transformation in the PTM. Within the PTM, cellular transformation induces infinite proliferation, altered morphology and enhanced invasiveness of malignant cells. Accordingly, various 3D MTSs and MTS-based approaches have been employed to model this process. Studies have shown that BC cell MTSs have increased malignant stemness and differentiation abilities with branching morphogenesis, loss of polarity, luminal filling and active cell invasion, ${ }^{35}$ which allow for assessment of therapeutic drugs, radiation and miRNA. ${ }^{36-38}$ In addition, the MTS applications can be combined with numerous bioreactors, including liquid overlay, spinning flask and gyratory rotation, hanging drop and suspension culture systems. ${ }^{39,40}$ The rotating wall vessel (RWV) has emerged in the microgravity milieu for constructing 3D cell tumour aggregates in which cells experience near-laminar flow conditions to survive in a suspension state in medium. ${ }^{41}$

Modelling immune-survival in the CM. MTS cultures of BC cells and alloimmune spleen cells was first used to demonstrate that immune cells are capable of penetrating tumour aggregates to kill interior malignant cells. ${ }^{42}$ However, devoid of stimulation by cytokines and monoclonal antibodies, cancer cells cultured in MTSs showed immune-resistance against NK cell-mediated death. ${ }^{43,44}$ Additionally, antigen presentation plays a vital role in immune attack, leading to the threshold of immune responses against cancer. ${ }^{45}$ This effect, which is mediated by antigenpresenting cells (APCs), such as dendritic cells, monocytes and macrophages, was re-established via co-culture of APCs in MTSs, which demonstrated a transition in morphology, invasiveness and differentiation that resulted in a decreased capacity for antigen presentation and promoted cancer malignancy. ${ }^{46-49}$

Modelling the BM. There are few reports on using MTS culture alone to model the BM, which is likely because this simplistic intercellular communication model fails to recreate the complex cancer-calcified matrix (bone) interplay. Thus, osteoblast-secreted matrices from MTSs have been used for modelling the BM. These matrices will be described in more detail in the "Tissue engineering construct" section. Here, MTSs of PC cells, osteosarcoma cells, osteoblast cells and stromal cells were generated for application in an RWV bioreactor, demonstrating that PC cells could induce permanent morphologic alterations in co-cultured osteoblasts and stromal cells, which stimulated the proliferation and invasiveness of PC cells. ${ }^{50}$ Compared with regular MTS culture, RWVs produce large batches of cell aggregates with a limited necrotic core ${ }^{51}$ and can be used to establish osteotropic tumours with areas of limited proliferation and decreased drug sensitivity. ${ }^{41,52}$ Moreover, unlike RWVs that minimise natural gravity, other bioreactors re-establish the natural forces within the BM. Using a specialised compartmentalised bioreactor, osteoblast MC3T3-E1 cells were co-cultured with BC MDA-MB-231 cells, and the results showed that less mature osteoblasts could bolster BC localisation more readily. Co-culture of $B C$ cells further induced activation of osteoblasts and osteoclasts with reduced expression of osteocalcin and increased IL-6 levels, resulting in remarkable bone matrix degradation. ${ }^{53,54}$ This biosystem was implemented in another specialised bioreactor that allowed for maturation of 3D multi-celllayer osteogenic tissue to study the metastatic interactions between cancer cells and bone ${ }^{55}$ (Fig. 3). BC cells tended to invade through the thick bone matrix after the early stages of metastasis, leading to matrix destruction. Mastro et al. ${ }^{55}$ showed that BC MDA-MB-231 GFP cells could invade thick osteoblastembedded ECM to form "Indian files", chains depicted as infiltrating lobular or metaplastic BCs. Osteoblasts were found to align in parallel with the cancer cells and exhibited increased inflammatory cytokine production. In addition, subsequent stages of invasive BC cells developed enhanced chemotaxis towards activated osteoclasts to form tumour colonies, accompanied by decreased osteoblastic tissue thickness and increased differentiation of osteoclast precursors. ${ }^{56,57}$ These bioreactors support MTS cultures (cancer cells, osteoblasts and osteoclasts) and thus contribute to the re-establishment of a "vicious cycle" for studying osteobiology and osteopathology in BM models of bone metastasis in vitro.

Tissue engineering constructs (TECs) and scaffolds TECs and scaffolds are the most widely used 3D approaches for modelling the microenvironments of bone metastasis because of their relatively available sources and convenient manipulation. ${ }^{58,59}$ Herein, scaffolds applied for TECs are based on natural materials or synthetic polymers, which have distinct advantages and disadvantages. Natural construct scaffolds are similar to natural ECM and possess inherent biocompatibility that is derived from the use of non-toxic substances. Synthetic constructs can be obtained from medical-grade purified molecules and manufactured under personalised guidelines. However, the drawbacks of natural and synthetic materials are obvious. Natural constructs have weak mechanical properties, uncontrollable degradability and betweenbatch inconsistency. Synthetic constructs carry the risk of loss of 
intrinsic bioactivity and modulus stiffness over the incubation period, leading to degradation of these compounds into toxic decomposition products. Therefore, it is vital to weigh both the advantages and disadvantages to establish the most suitable 3D TEC models for bone metastasis studies; this effort has led to the combination of natural molecules and synthetic scaffolds, which is a promising strategy for modelling bone metastasis.

Modelling cancer-related angiogenesis in the PTM. Natural materials, such as collagen, fibrin and laminin-rich basement membrane extract (BME/Matrigel), which originate from the ECM, have advantages in providing natural sites for cell attachment and signalling conduction to promote endothelial cell proliferation, migration and formation into capillary-like microstructures. ${ }^{60-63}$ This type of ECM-derived biomaterial has long been the most widely used approach in cancer-related angiogenesis studies. For instance, osteotropic BC MCF-7 cells cultured in 3D Matrigel have increased expression of VEGF, bFGF and IL-8, which are the main mediators of endothelial cell recruitment and differentiation and thus promote angiogenesis. ${ }^{64}$ Culturing endothelial cells on collagen matrices with cancer cells and fibroblasts leads to the formation of branching microvasculature, indicating that cancer cells and fibroblasts embedded in collagen can trigger migration and differentiation of endothelial cells with elevated expression of VEGF. ${ }^{60,65}$

Recently, the utilisation of semi-synthetic, naturally derived, protein-based hydrogels, such as alginate- and collagen-based hydrogels, has surpassed the use of wholly synthetic scaffolds, such as poly(lactide-co-glycolide) (PLG) and poly(lactide-coglycolide acid) (PLGA), in modelling angiogenesis since synthetic scaffolds alone lack bioactive growth factors. Thus, co-culture of osteotropic PC cells and endothelial cells on hydrogel decorated with IKVAV and GFOGER peptides was found to lead to increased vascular cell infiltration with significant blood tube outgrowth. ${ }^{66,67}$ Poly(ethylene)glycol (PEG)-based hydrogel-supported LC cells induce the release of proangiogenic TGF- $\beta$ to stimulate the neovasculature of endothelial cells, resulting in further significant tumour progression compared to hydrogels without vascular cells. ${ }^{68}$ Moreover, co-culture with endothelial cells or embryoid bodies contributed to the directional migration of endothelial cells towards the tumour, leading to the establishment of a tubular structure and luminal network. ${ }^{69,70}$ Another significant 3D biosystem is the combination of a human arterial ring (hAR) with Matrigel in which PC LNCaP cells facilitate angiogenesis by stimulating the release of proangiogenic factors from the hAR to sustain angiogenesis in the absence of an exogenous stimulus. $^{71}$

Modelling cellular transformation in the PTM. Using natural matrices that mimic the structural and mechanical properties of the ECM, Oyanagi et al. ${ }^{72}$ found that LC A549 cells treated with TGF- $\beta$ in a $3 D$ collagen matrix had EMT traits, including extended microtubule-based protrusions and increased migration ability. ${ }^{72}$ Additionally, BC cells cultured in a collagen matrix developed irregular cellular polarity, increased cisplatinum resistance and enhanced metastasis ability, which can be attributed to the altered expression of integrins, proangiogenic factors and EMT proteins in 3D cultures. ${ }^{40,73}$ Fibroblast-derived matrices produced by cancer-associated fibroblasts have also been used to culture osteotropic cancer cells, inducing a significant change in cell morphology, proliferation and invasiveness compared to 2D cultures. $^{74}$ In addition, artificial synthetic scaffolds, such as hyaluronic acid (HA) hydrogels, can support a finger-like architecture of PC cells, allowing for quantification of cell size, shape and convergence, which support the metastatic ability of PC cells. ${ }^{75}$ Additionally, PLGA and poly( $\varepsilon$-caprolactone) (PCL) scaffolds can support the 3D culture of malignant cells, enabling investigation of microenvironment stimuli acting on these cells, including inhibition of proliferation and invasion with cytotoxic drugs. ${ }^{76,77}$ PC cells cultured in a chitosan-HA matrix developed stem-like features and EMT attributes compared to those in a 2D system, revealing that HA likely promotes the metastasis, EMT and drug resistance of PC cells, followed by the activation of downstream signalling involved in cancer malignancy. ${ }^{78}$

Modelling immune-survival in the CM. Considering the actual purposes of CM modelling, various natural or synthetic scaffolds were combined with MTSs. A 3D spheroid on collagen gel gave cancer cells the ability to promote tumour-associated macrophage (TAM) transition, ${ }^{79}$ providing an immune-regulatory potential for evaluating the in vivo-like immunotherapeutic effects of immune cells compared with $2 \mathrm{D}$ cultures. ${ }^{80,81}$ When cultured on a 3D chitosan-alginate scaffold, BC cells expressed IFN- $\gamma$ and CCL21 cytokines to induce the recruitment and infiltration of $T$ cells into a MTS, providing a promising CM model for assessing T-cell function. $^{82}$

Modelling directional migration in the CM. Collagen-based scaffolds supported directional metastasis of $B C$ cells with invasive phenotypes compared to Matrigel or RADA16 peptide scaffolds, which could be enhanced by the increased collagen density. ${ }^{83-85}$ A bovine serum albumin (BSA) scaffold with fibronectin (FN) showed desirable BC cell adhesion and migration compared with the BSA scaffold alone. ${ }^{86}$

Modelling the BM. Notably, since osteoblast differentiation and mineralisation leads to the formation of mature bone, ${ }^{87}$ it is feasible to use osteoblasts to model 3D osteoblastic matrices for cancer cells in the BM. Mineralised ECM released from human osteoblasts supports cellular progression of metastatic BC cells by establishing potent attachment forces between BC cells and osteoblasts, which are mediated by the production of $\beta_{1}$ integrin, osteopontin and matrix-metalloproteinase (MMP) 2 and 9 in metastatic BC cells. ${ }^{88}$ Similarly, another decellularized osteoblastic mineralized matrix indicated that a collagen-l-rich fibril matrix with various ECM proteins could provide a compatible microenvironment for PC cells to induce adhesion, proliferation and metastasis. ${ }^{89}$ Nevertheless, such models of osteoblast-secreted matrices are considered difficult to manufacture on a large scale and have insufficient stiffness, resulting in formidable challenges for translational applications. A 3D approach for engineering osteoblastic bone tissue was developed, combining the advantages of osteoblastic matrices with a medical-grade polymer system. Polycaprolactone-tricalcium phosphate (PCL-TCP) scaffolds wrapped with induced mineralized osteoblast sheets were devised for co-culture with PC cells, revealing the compatible colonization of PC cells onto engineered bone with metastasis-associated molecules, such as MMPs, prostate specific antigen (PSA) and steroidogenic enzymes. ${ }^{90}$ When PC cells were pre-embedded in hydrogel to synthesise the PCL-TCP scaffold, engineered bone decreased the ingrowth of PC cells. Importantly, PC cells produced osteomimetic cell phenotypes, as exemplified by upregulation of bone and vasculature markers and activation of TGF- $\beta$ downstream genes. $^{91}$

Although numerous 3D osteoblastic approaches have been used to model osseous or osseous-like scaffolds of the BM, natural and synthetic TECs can also recreate the osteomimetic response of osteotropic cells with other cell types that constitute the bone microenvironment. For example, various 3D collagen gels (collagen-based glycosaminoglycan or nanohydroxyapatite) have been used to simulate biological intercellular events between metastatic cells and bone marrow-derived cell types, such as adipocytes, mesenchymal cells and endothelial cells, to study the directional bone-homing and proliferative effects of malignant cells. ${ }^{92-94}$ Fitzgerald et al. ${ }^{94}$ demonstrated that three 


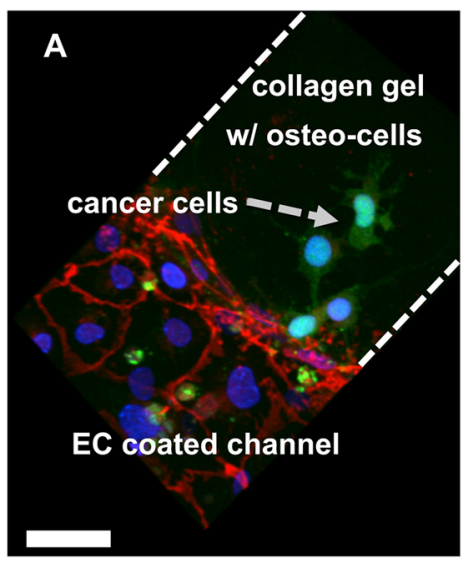

B
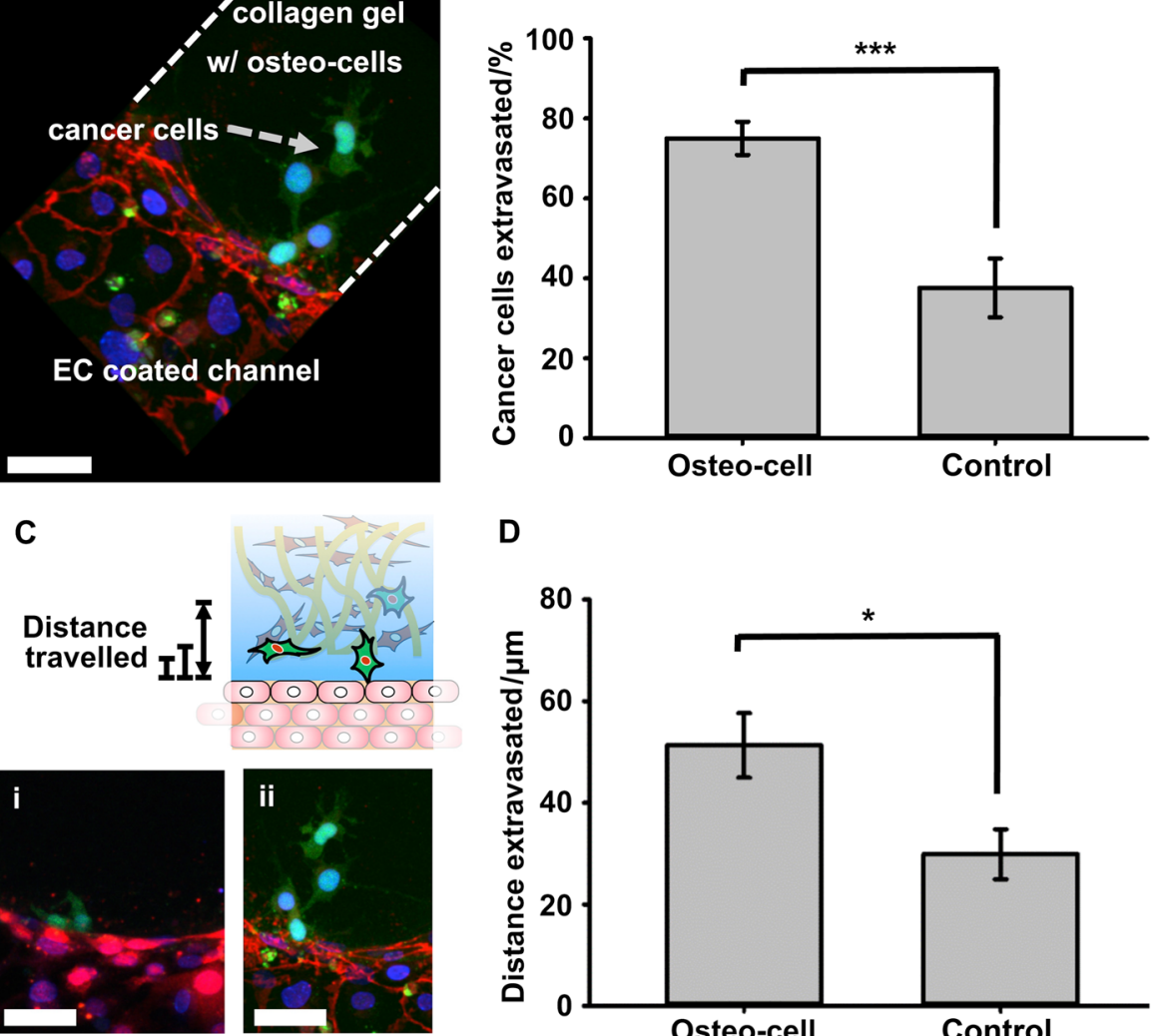

D

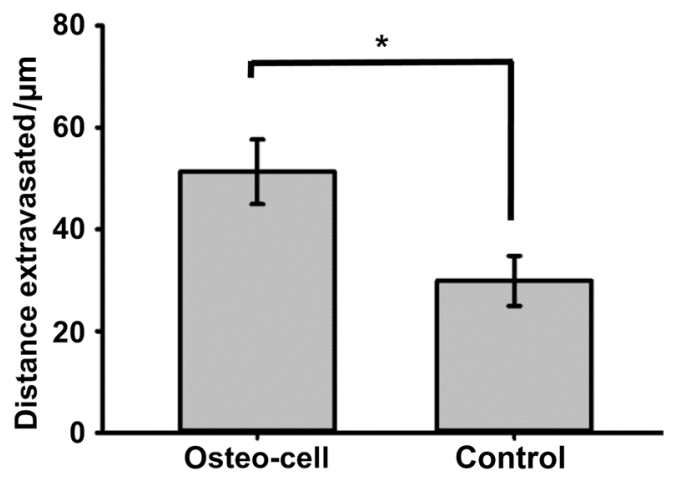

Fig. 4 Extravasation of cancer cells into the collagen gel matrix with and without osteo-differentiated hBM-MSCs (labelled as osteo-cells). a Three-dimensional confocal reconstruction shows MDA-MB-231 cancer cells (GFP) transmigrated across the endothelial monolayer into the collagen gel containing osteo-differentiated hBM-MSCs. VE-cadherin (red) and DAPI (nuclei, blue) staining. b Average percentage of extravasated cancer cells was significantly higher $\left({ }^{* *} P<0.005\right)$ in the collagen gel with osteo-differentiated hBM-MSCs. c Projected images show extravasated cancer cells (GFP) travelled farther into the osteo-cell conditioned microenvironment (ii) compared to the collagen gel-only matrix (i). Cells were stained with (i) DAPI (nuclei, blue) and (ii) VE-cadherin (red) + DAPI (nuclei, blue). HUVECs were RFP labeled. d Average distance travelled by extravasated cells into the gel matrix increased significantly in osteo-cell conditioned microenvironment $(* P<0.05)$. Scale bars represent $50 \mu \mathrm{m}$. Reprinted with permission from ref. ${ }^{14}$ Copyright (2014) Elsevier Publishing Group

types of collagen-based scaffolds (ColGAG, S200 and S500) remained structurally stable after 14 days of PC cell co-culture, indicating the potential application of collagen-based ColGAG, S200 and S500 scaffolds for PC implantation up to 14 days. Moreover, transplantation of bone marrow stromal cells (BMSCs) in silk scaffolds and BMP-2 stimulation of mouse mammary pads demonstrated the bone-targeting effects of metastatic BC cells in vivo. ${ }^{95,96}$ BC $4 \mathrm{~T} 1$ cells cultured in $3 \mathrm{D}$ a collagenglycosaminoglycan scaffold developed mineralization sites with increased levels of collagen-I and bone sialoprotein when stimulated with exogenous phosphate and BMP-2. ${ }^{97}$ Stromal and endothelial cells were used on the fibrin scaffold to develop a 3D BM for MM bone metastasis, recapitulating the 3D metastatic bone niche for MM cells. ${ }^{98}$ Mesenchymal progenitor cells were transplanted onto a tubular composite scaffold supplemented with BMP-7 for PC bone metastasis, demonstrating the morphogenesis of physiological bone tissues and the directional migration of PC cells to humanised bone tissues with cancer-induced bone lesions. ${ }^{99}$

Microfluidic systems

In vivo, osteotropic cancer cells experience a continuous fluidic microenvironment in the PTM, CM and BM. Hence, it is important to consider the fluid stresses that cancer cells withstand when modelling metastatic microenvironments. Microfluidic systems that provide high-throughput and high-content approaches ${ }^{100}$ have received significant attention in the $3 \mathrm{D}$ recapitulation of cancer bone metastasis. Such systems allow for effective highresolution and high-sensitivity analysis with a small number of cells and reagents to create in vivo-like 3D flowing conditions in vitro.

Modelling cancer-angiogenesis in the PTM. Three-dimensional bioengineered microfluidic scaffolds have well-proportioned fluid stress, leading to balanced dissemination of cancer cells with supportive cells within uniform-sized cell spheroids ${ }^{101}$ that can maintain malignant angiogenesis in the PTM. One example is the co-culture of BC cells with endothelial cells in a microfluidic system in which low fluidic conditions contributed to the convergence of endothelium with a functional microchannel lumen and enhanced cancer malignancy. ${ }^{102}$

Modelling cellular transformation in the PTM. Cancer cells cultured in a microfluidic biosystem developed spheroid cell aggregates exhibiting decreased levels of epithelial markers, such as CD326, and elevated expression of mesenchymal markers, such as $\mathrm{N}$ cadherin, vimentin and fibronectin, compared with cells cultured in the $2 \mathrm{D}$ bio-platform. ${ }^{103}$ Additionally, a gel-free nanoscaffold provided LC cells with a 3D milieu for TGF- $\beta$-induced EMT transformation morphologically and genetically, ${ }^{104}$ indicating 
potential re-establishment of the EMT process using a micropattern approach.

Modelling directional migration in the CM. The 3D approaches for the $\mathrm{CM}$ also require recapitulation of the kinesiology of osteotropic cancer cells, which can generally be classified as transendothelial movements (intravasation and extravasation) and directional migration within the circulatory system. Penetration through endothelium depends on the active interaction between cancer cells and stromal cells, and the directional migration and invasion of malignant cells rely on the chemoattraction of homing sites. ${ }^{105,106}$

To date, researchers have primarily taken advantage of microfluidic systems to establish dosage gradients of chemokines and to simulate the shear stress and interstitial flow that the endothelial network imposes during transendothelial migration to model the dynamic bioactivity of cancer cells in the CM. ${ }^{75}$ The hydrodynamics of a microfluidic chip enable assessment of tumour cell alterations at morphological, genetic and protein levels related to membrane rearrangement and subsequent migratory activity. ${ }^{107,108}$ Cancer cells can transmigrate through the endothelial monolayer into the collagen gel within the microfluidic platform, showing tightly regulated and wellmodelled extravasation of cancer cells, as evidenced by the ability to target the regulation of CXCL12, epidermal growth factor (EGF), interstitial flow, cell morphology and matrix stiffness. ${ }^{109-111}$ Additionally, a cell-based microfluidic chip was devised in which both the intravasation and extravasation processes in metastasis could simultaneously reappear, demonstrating the detachment of malignant cells from the Matrigel matrix under sheer stress in the intravasation chamber, followed by the attachment to adhesion molecules expressed by endothelial cells in the extravasation chamber. $^{112}$

Modelling the BM. A microfluidic device was applied to modelling the BM because this device can mimic the bone milieu for investigating dynamic cancer metastasis. To construct an organ-specific 3D microfluidic approach for osteotropic cell metastasis, primary and mineralized bone mesenchymal stem cells (BMSCs) and endothelial cells were tri-cultured, leading to the establishment of a vascularises bone-like microenvironment for studying the extravasation rate and microvascular permeability of BC cells. ${ }^{113}$ Similarly, a vascularises microfluidic system of BMSCs and endothelial cells was devised, followed by the subsequent introduction of BC MDA-MB-231 cells into vessel-like channels, resulting in a viable formation of micrometastases of extravasated cancer cells in the attractive matrix ${ }^{114}$ (Fig. 4). Moreover, by combining the microfluidic bio-platform with tricultured cell spheroids comprising osteoblasts, endothelial cells and PC cells, this flowing chip enabled real-time monitoring of each spheroid and the interior PC cells, indicating the curbed proliferation of PC cells without jeopardising the cell viability. This system could be employed to more accurately model the in vivo growth patterns of malignant cells within the metastatic BM. $^{101}$

\section{Three-dimensional bioprinting}

Recent progress in 3D bioprinting has triggered substantial innovations in the construction of sophisticated 3D functionalized living tissues and organs, including bones, ${ }^{115-117}$ and there is increasing interest in re-establishment of the dynamic microenvironments of bone metastasis. The use of bio-inks, which consist of cell mixtures in the form of compatible gel-like material, is expected to be used for depositing and generating 3D scaffolds that mimic spatial metastatic microenvironments at high resolution. ${ }^{118}$ However, compared with other 3D approaches commonly used for engineering dynamic microenvironments, there are limited reports on the use of 3D bioprinting to evaluate cancer bone metastasis, and bioprinting might be an attractive realm for future research and translation.

Modelling cancer-angiogenesis in the PTM. Because 3D bioprinting fibres embedded in hydrogels can generate microvessels, patterning cells and biomaterials for cancer-angiogenesis in PTM has received much attention. ${ }^{119}$ In one study, a sacrificial template was deposited with a bioprinter in a random pattern similar to the host vasculature network, followed by placement of the cast hydrogel around the template. After removal of the template, there were porous microchannels within the hydrogel. Along with these microchannels, endothelial cells could be internalised to faithfully form a functionalized vasculature system. Based on these results, a microchannel system with human umbilical vein endothelial cells (HUVECs) and seeded with MTSs showed that MTSs were remodelled and exhibited potential angiogenesis. ${ }^{120}$

Modelling cellular transformation in the PTM. By co-culturing LC cells with agarose and alginate, which served as scaffold materials to mimic the growth milieu, 3D bioprinting allowed for desirable proliferation of LC cells, which were able to migrate and invade into the adjacent scaffold. ${ }^{121}$ Furthermore, the in vivo orientation between stromal cells and cancer cells could be recapitulated via $3 \mathrm{D}$ bioprinting in vitro by depositing cell suspensions onto the basement in a predefined pattern, revealing a feasible co-culture platform that was amenable to controlling cell droplet size, density and malignant morphology. The cells in this system had a more metastatic phenotype than did those in 2D cultures. ${ }^{122,123}$ Additionally, BC cells cultured in a custom-built bioprinting platform demonstrated more in vivo-like and uniform cellular spheroids in situ on a hydrogel substrate, representing a controllable and high-throughput approach for modelling PTM. ${ }^{124}$

Modelling immune-survival in the CM. It was demonstrated that a co-extrusion bioprinting model could be used to study the interaction between BC cells and macrophages, contributing to the development of paracrine cycle-stimulated migration and extravasation of BC cells. ${ }^{125}$ The results further showed that macrophages could escape from microvessels to communicate with $\mathrm{BC}$ cells, providing a convenient model for studying immunesurvival of cancer cells in the CM.

Modelling directional migration in the CM. Three-dimensional bioprinting is advantageous for providing a CM matrix that supports the migration of osteotropic cells during bone metastasis. A honeycomb structure in a hydrogel was created with 3D bioprinting to simulate the vasculature in vitro for evaluating the migration of cancer cells, which showed that a reduced width of bioprinted capillaries increased the migratory velocity of cancer cells. ${ }^{126}$ In addition to the vessel diameter, a bioprinted PEG scaffold showed that substrate stiffness and cell morphology significantly impacted the migration speed of malignant cells. ${ }^{127}$

Modelling the BM. Comparison of typical 3D natural and synthetic scaffolds in terms of the substrate modulus and pore size indicates that $3 \mathrm{D}$ bioprinting produces more in vivo-like trabecular bone for modelling the BM in bone metastasis. A template-fused deposition modelling strategy was used to generate a tuneable porous matrix with skeletal parameters similar to in vivo cancellous bone. ${ }^{128}$ Furthermore, when cocultured with BMSCs, this bioprinting system enabled recapitulation of enhanced osteogenesis with increased matrix stiffness and decreased pore diameter, which successfully modelled the BM during bone metastasis. Additionally, BMSCs or osteoblasts encapsulated in gelatine methacrylate (GeIMA) and polyethylene glycol hydrogel with nanocrystalline HA were used to constitute the bone matrix for bioprinting. The subsequent co-culturing of BC cells potentiated cancer cell ingrowth and enhanced the 


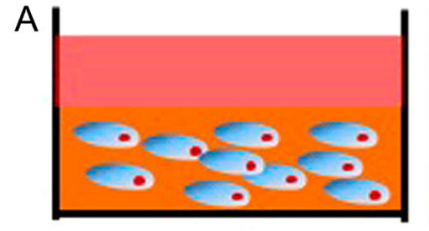

Mono-culture of osteoblasts
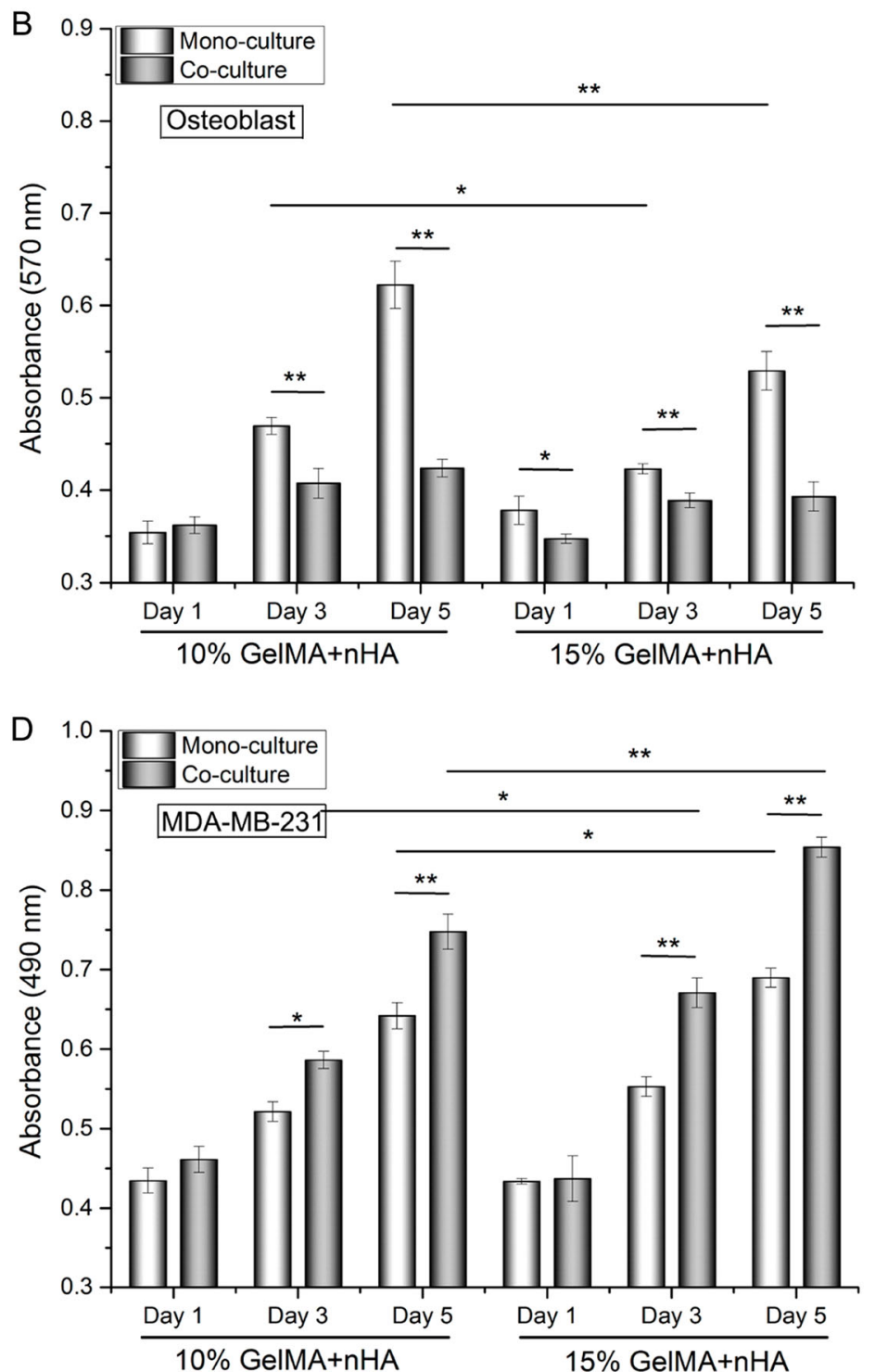
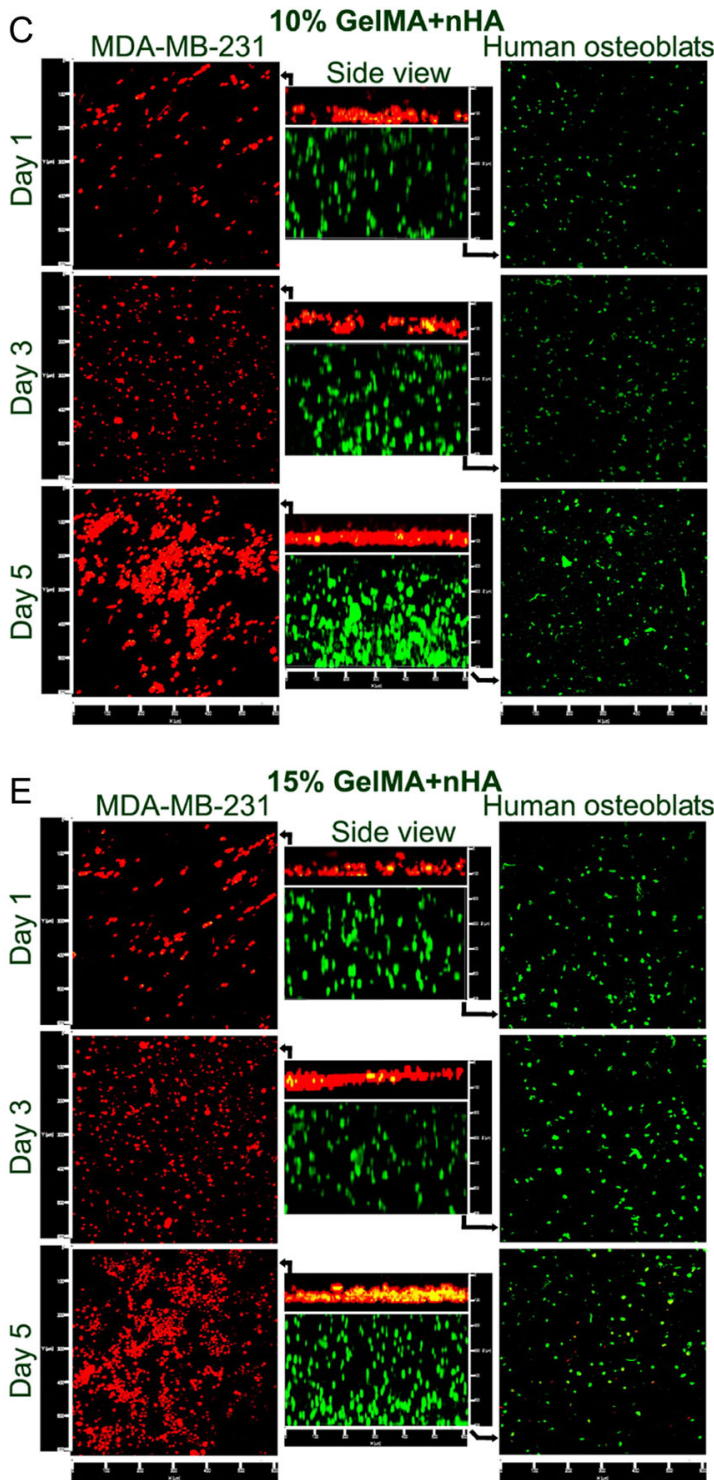

Fig. 5 a Schematic diagram of osteoblasts and BrCa cells mono- and co-cultured in the 3D bioprinted matrix. Proliferation of $\mathbf{b}$ osteoblasts and $\mathbf{d}$ BrCa cells mono- and co-cultured in the $3 \mathrm{D}$ bioprinted matrix after 1,3 and 5 days. ${ }^{*} P<0.05,{ }^{* *} P<0.01$. c, e Confocal micrographs of osteoblasts and $\mathrm{BrCa}$ cells co-cultured in the 3D bioprinted matrix after 1, 3 and 5 days. The middle columns in c, e represent the crosssectional views. Osteoblasts and BrCa cells were stained by Cell Tracker Green CMFDA dye (green) and Orange CMTMR dye (red), respectively. Reprinted with permission from ref. ${ }^{129}$ Copyright (2016) American Chemical Socie1ty

expression of VEGF and IL-8 due to stimulation of BMSCs or osteoblasts. ${ }^{129,130}$ Zhou et al. ${ }^{131}$ showed that the proliferation of BC cells was enhanced due to osteoblast co-culture, while the growth of osteoblasts was inhibited because of BC co-culture, especially in 3D bioprinting scaffolds $(10 \% / 15 \%$ GelMA $+n H A)$ (Fig. 5). Moreover, BC cells formed spheroids with potent metastatic characteristics. ${ }^{131}$ However, this effect was alternatively followed by an inhibition of osteoblastic mineralization in 3D bioprinted cultures.

\section{CONCLUSION AND FUTURE PERSPECTIVES}

Three-dimensional engineering approaches provide controllable and representative toolkits to model the dynamic 
microenvironments of bone metastasis with the incorporation of functionalized cells, ECM, growth cytokines and other biochemical stimuli. These models allow for a reliable recapitulation of cancer metastatic microenvironments and facilitate the evaluation of cell-cell and cell-ECM crosstalk and thus can aid in elucidating malignant metastatic mechanisms and will enable the development of novel therapeutic strategies. ${ }^{132}$

However, major challenges and limitations remain. First, none of the current models recapitulates the entire process of metastasis in a single culture system. Notably, osteotropic cancer cells that metastasise from the primary site to distant bone undergo a sequential and closely related multistep process, and the cellular events cannot be separated. Second, advances in 3D cultures mainly employ immortalized malignant cell lines while neglecting to introduce patient-derived cancer cells. The use of personalised cells rather than cell lines could aid in the development of customized therapies and would guide the treatment of patients with bone metastasis more effectively. ${ }^{19,133,134}$ Third, unlike the spheroids of MTSs in vitro, in vivo tumours tend to establish various 3D appearances, such as cauliflower-like, ulcerative and irregular-shaped forms. This discrepancy is of vital significance because the stereo-architecture of tumours remarkably affects osteotropic cancers in terms of oxygen diffusion, cell adhesion, and drug sensitivity. Fourth, because each 3D approach has its own merits and demerits, a potential future trend is to combine diverse platforms to mimic extensive metastatic microenvironments. For instance, a microfluidic system was enhanced with 3D bioprinting to fabricate specialised, customized and flowing devices suitable for particular applications. ${ }^{135}$ Lastly, although osteocytes play significant roles during bone metastasis, reports concerning the combination of osteocytes with dynamic 3D approaches to model the BM of bone metastasis are rare. Indeed, this interesting practice requires further study. Overall, despite the inspiring advances in engineering 3D approaches to model the dynamic microenvironments of bone metastasis, there is significant room to improve 3D approaches for a better understanding of the underlying mechanisms involved in bone metastasis.

\section{ACKNOWLEDGEMENTS}

This work was funded by National Natural Science Foundation of China (81672205), National Key R\&D Programme (2016YFC1102100) and Innovation Programme for Ph.D Students in Shanghai Jiao Tong University School of Medicine (BXJ201729).

\section{ADDITIONAL INFORMATION}

Competing interests: The authors declare no competing financial interests.

\section{REFERENCES}

1. Yan, W. et al. Suppressive effects of plumbagin on invasion and migration of breast cancer cells via the inhibition of STAT3 signaling and down-regulation of inflammatory cytokine expressions. Bone Res. 1, 362-370 (2013).

2. Baier, S. R. \& Wan, Y. MicroRNA exert macro-effects on cancer bone metastasis. Curr. Osteoporos. Rep. 14, 163-169 (2016).

3. $\mathrm{Wu}, \mathrm{B}$. et al. Atypical skeletal manifestations of rickets in a familial hypocalciuric hypercalcemia patient. Bone Res. 5, 17001 (2017).

4. Grinnell, F. Fibroblast biology in three-dimensional collagen matrices. Trends Cell Biol. 13, 264-269 (2003).

5. Peela, N. et al. A three dimensional micropatterned tumor model for breast cancer cell migration studies. Biomaterials 81, 72-83 (2016).

6. Chen, S. H. et al. PLGA/TCP composite scaffold incorporating bioactive phytomolecule icaritin for enhancement of bone defect repair in rabbits. Acta Biomater. 9, 6711-6722 (2013).

7. Fazilaty, H. \& Mehdipour, P. Genetics of breast cancer bone metastasis: a sequential multistep pattern. Clin. Exp. Metastas-. 31, 595-612 (2014).

8. Paget, S. The distribution of secondary growths in cancer of the breast. 1889. Cancer Metastas-. Rev. 8, 98-101 (1989).
9. Douglas Hanahan, J. F. Patterns and emerging mechanisms of the. Cell 86, 353-364 (1996).

10. Auguste, P. et al. Molecular mechanisms of tumor vascularization. Crit. Rev. Oncol. Hematol. 54, 53-61 (2005).

11. Thiery, J. P. \& Sleeman, J. P. Complex networks orchestrate epithelialmesenchymal transitions. Nat. Rev. Mol. Cell Biol. 7, 131-142 (2006).

12. Chambers, A. F., Groom, A. C. \& MacDonald, I. C. Dissemination and growth of cancer cells in metastatic sites. Nat. Rev. Cancer 2, 563-572 (2002).

13. Adjei, I. M. \& Blanka, S. Modulation of the tumor microenvironment for cancer treatment: a biomaterials approach. J. Func. Biomater. 6, 81-103 (2015).

14. Douma, S. et al. Suppression of anoikis and induction of metastasis by the neurotrophic receptor TrkB. Nature 430, 1034-1039 (2004).

15. Weidle, U. H. et al. Molecular mechanisms of bone metastasis. Cancer Genom. Proteom. 13, 1-12 (2016).

16. Giancotti, F. G. Mechanisms governing metastatic dormancy and reactivation. Cell 155, 750-764 (2013).

17. Bonewald, L. F. The amazing osteocyte. J. Bone Miner. Res. 26, 229-238 (2011).

18. Nakashima, T. et al. Evidence for osteocyte regulation of bone homeostasis through RANKL expression. Nat. Med. 17, 1231-1234 (2011).

19. Qiao, H. et al. Targeting osteocytes to attenuate early breast cancer bone metastasis by theranostic upconversion nanoparticles with responsive plumbagin release. ACS Nano 11, 7259-7273 (2017).

20. Giuliani, N. et al. Increased osteocyte death in multiple myeloma patients: role in myeloma-induced osteoclast formation. Leukemia 26, 1391-1401 (2012).

21. Sottnik, J. L. et al. Tumor-induced pressure in the bone microenvironment causes osteocytes to promote the growth of prostate cancer bone metastases. Cancer Res. 75, 2151-2158 (2015).

22. Hensel, J. \& Thalmann, G. N. Biology of bone metastases in prostate cancer. Urology 92, 6-13 (2016).

23. Buijs, J. T. \& van der Pluijm, G. Osteotropic cancers: from primary tumor to bone. Cancer Lett. 273, 177-193 (2009).

24. Hanahan, D. \& Weinberg, R. A. Hallmarks of cancer: the next generation. Cell 144, 646-674 (2011).

25. Alemany-Ribes, M. \& Semino, C. E. Bioengineering 3D environments for cancer models. Adv. Drug Deliv. Rev. 79-80, 40-49 (2014).

26. Salamanna, F. et al. A systematic review on in vitro 3D bone metastases models: A new horizon to recapitulate the native clinical scenario? Oncotarget 7 , 44803-44820 (2016).

27. Verjans, E. T. et al. Three-dimensional cell culture models for anticancer drug screening: worth the effort? J. Cell. Physiol. 233, 2993-3003 (2017).

28. Halfter K., \& Mayer B. Bringing 3D tumor models to the clinic-predictive value for personalized medicine. Biotechnol. J. 12, 1600295 (2017).

29. Santo V. E. et al. Drug screening in $3 D$ in vitro tumor models: overcoming current pitfalls of efficacy read-outs. Biotechnol. J. 12, 1600505 (2017).

30. Ravi, M., Ramesh, A. \& Pattabhi, A. Contributions of 3D cell cultures for cancer research. J. Cell. Physiol. 232, 2679-2697 (2017).

31. Fong, E. L. et al. Heralding a new paradigm in 3D tumor modeling. Biomaterials 108, 197-213 (2016).

32. Duval, K. et al. Modeling physiological events in 2D vs. 3D cell culture. Physiol 32, 266-277 (2017).

33. Sutherland, R. M. et al. A multi-component radiation survival curve using an in vitro tumour model. Int. J. Radiat. Biol. Relat. Stud. Phys. Chem. Med. 18, 491-495 (1970).

34. Grimes, D. R. et al. A method for estimating the oxygen consumption rate in multicellular tumour spheroids. J. R. Soc. Interface 11, 20131124 (2014).

35. Nelson, C. M., Inman, J. L. \& Bissell, M. J. Three-dimensional lithographically defined organotypic tissue arrays for quantitative analysis of morphogenesis and neoplastic progression. Nat. Protoc. 3, 674-678 (2008).

36. Boo, L. et al. MiRNA transcriptome profiling of spheroid-enriched cells with cancer stem cell properties in human breast MCF-7 cell line. Int. J. Biol. Sci. 12, 427-445 (2016).

37. Rouhani, M. et al. Lithium increases radiosensitivity by abrogating DNA repair in breast cancer spheroid culture. Arch. Iran. Med. 17, 352-360 (2014).

38. Ray, A., Vasudevan, S. \& Sengupta, S. 6-Shogaol inhibits breast cancer cells and stem cell-like spheroids by modulation of Notch signaling pathway and induction of autophagic cell death. PLOS ONE 10, e0137614 (2015).

39. Page, H., Flood, P. \& Reynaud, E. G. Three-dimensional tissue cultures: current trends and beyond. Cell. Tissue Res. 352, 123-131 (2013).

40. Kim, J. B. Three-dimensional tissue culture models in cancer biology. Semin. Cancer Biol. 15, 365-377 (2005).

41. Becker, J. L. \& Souza, G. R. Using space-based investigations to inform cancer research on Earth. Nat. Rev. Cancer 13, 315-327 (2013).

42. Sutherland, R. M., MacDonald, H. R. \& Howell, R. L. Multicellular spheroids: a new model target for in vitro studies of immunity to solid tumor allografts. J. Natl. Cancer Inst. 58, 1849-1853 (1977). 
43. Hoffmann, T. K. et al. A novel mechanism for anti-EGFR antibody action involves chemokine-mediated leukocyte infiltration. Int. J. Cancer 124, 2589-2596 (2009).

44. Holmes, T. D. et al. A human NK cell activation/inhibition threshold allows small changes in the target cell surface phenotype to dramatically alter susceptibility to NK cells. J. Immunol. 186, 1538-1545 (2011).

45. van Kasteren, S. I. et al. Chemical biology of antigen presentation by MHC molecules. Curr. Opin. Immunol. 26, 21-31 (2014).

46. Dietl, K. et al. Lactic acid and acidification inhibit TNF secretion and glycolysis of human monocytes. J. Immunol. 184, 1200-1209 (2010).

47. Hauptmann, S. et al. Macrophages and multicellular tumor spheroids in coculture: a three-dimensional model to study tumor-host interactions. Evidence for macrophage-mediated tumor cell proliferation and migration. Am. J. Pathol. 143, 1406-1415 (1993).

48. Gottfried, E. et al. Tumor-derived lactic acid modulates dendritic cell activation and antigen expression. Blood 107, 2013-2021 (2006).

49. Kim, T. E. et al. Three-dimensional culture and interaction of cancer cells and dendritic cells in an electrospun nano-submicron hybrid fibrous scaffold. Int. J. Nanomed. 11, 823-835 (2016).

50. Sung, S. Y. et al. Coevolution of prostate cancer and bone stroma in threedimensional coculture: implications for cancer growth and metastasis. Cancer Res. 68, 9996-10003 (2008).

51. Hammond, T. G. \& Hammond, J. M. Optimized suspension culture: the rotatingwall vessel. Am. J. Physiol. 281, F12-F25 (2001).

52. Kaur, P. et al. Human breast cancer histoid: an in vitro 3-dimensional co-culture model that mimics breast cancer tissue. J. Histochem. 59, 1087-1100 (2011).

53. Krishnan, V. et al. Dynamic interaction between breast cancer cells and osteoblastic tissue: comparison of two- and three-dimensional cultures. J. Cell. Physiol. 226, 2150-2158 (2011).

54. Dhurjati, R. et al. Metastatic breast cancer cells colonize and degrade threedimensional osteoblastic tissue in vitro. Clin. Exp. Metastas-. 25, 741-752 (2008).

55. Mastro, A. M. \& Vogler, E. A. A three-dimensional osteogenic tissue model for the study of metastatic tumor cell interactions with bone. Cancer Res. 69, 4097-4100 (2009).

56. Krishnan, V. et al. In vitro mimics of bone remodeling and the vicious cycle of cancer in bone. J. Cell. Physiol. 229, 453-462 (2014).

57. Krishnan, V., Vogler, E. A. \& Mastro, A. M. Three-dimensional in vitro model to study osteobiology and ssteopathology. J. Cell. Biochem. 116, 2715-2723 (2015).

58. Liu, M. et al. Injectable hydrogels for cartilage and bone tissue engineering. Bone Res. 5, 17014 (2017).

59. Yi, H. et al. Recent advances in nano scaffolds for bone repair. Bone Res. 4, 16050 (2016).

60. Velazquez, O. C. et al. Fibroblast-dependent differentiation of human microvascular endothelial cells into capillary-like three-dimensional networks. Faseb. J. 16, 1316-1318 (2002).

61. Feng, X. et al. Fibrin and collagen differentially but synergistically regulate sprout angiogenesis of human dermal microvascular endothelial cells in 3dimensional matrix. Int. J. Cell Biol. 2013, 231279 (2013).

62. Benton, G. et al. Matrigel: from discovery and ECM mimicry to assays and models for cancer research. Adv. Drug Deliv. Rev. 79-80, 3-18 (2014).

63. Correa de Sampaio, P. et al. A heterogeneous in vitro three dimensional model of tumour-stroma interactions regulating sprouting angiogenesis. PLOS ONE 7, e30753 (2012).

64. Fischbach, C. et al. Engineering tumors with 3D scaffolds. Nat. Methods 4 855-860 (2007)

65. Kimlin, L. C., Casagrande, G. \& Virador, V. M. In vitro three-dimensional (3D) models in cancer research: an update. Mol. Carcinog. 52, 167-182 (2013).

66. Tsurkan, M. V. et al. Defined polymer-peptide conjugates to form cell-instructive starPEG-heparin matrices in situ. Adv. Mater. 25, 2606-2610 (2013).

67. Taubenberger, A. V. et al. 3D extracellular matrix interactions modulate tumour cell growth, invasion and angiogenesis in engineered tumour microenvironments. Acta Biomater. 36, 73-85 (2016).

68. Roudsari, L. C. et al. A 3D Poly(ethylene glycol)-based tumor angiogenesis model to study the influence of vascular cells on lung tumor cell behavior. Sci. Rep. $\mathbf{6}$, 32726 (2016).

69. Wartenberg, M. et al. Inhibition of tumor-induced angiogenesis and matrixmetalloproteinase expression in confrontation cultures of embryoid bodies and tumor spheroids by plant ingredients used in traditional chinese medicine. Lab. Invest. 83, 87-98 (2003)

70. Timmins, N. E., Dietmair, S. \& Nielsen, L. K. Hanging-drop multicellular spheroids as a model of tumour angiogenesis. Angiogenesis 7, 97-103 (2004).

71. Seano, G. et al. Modeling human tumor angiogenesis in a three-dimensional culture system. Blood 121, e129-e137 (2013).

72. Oyanagi, J. et al. Epithelial-mesenchymal transition stimulates human cancer cells to extend microtubule-based invasive protrusions and suppresses cell growth in collagen gel. PLOS ONE 7, e53209 (2012).
73. Chen, L. et al. The enhancement of cancer stem cell properties of MCF-7 cells in $3 \mathrm{D}$ collagen scaffolds for modeling of cancer and anti-cancer drugs. Biomaterials 33, 1437-1444 (2012).

74. Serebriiskii, I. et al. Fibroblast-derived 3D matrix differentially regulates the growth and drug-responsiveness of human cancer cells. Matrix Biol. 27, 573-585 (2008).

75. Xu, X., Farach-Carson, M. C. \& Jia, X. Three-dimensional in vitro tumor models for cancer research and drug evaluation. Biotechnol. Adv. 32, 1256-1268 (2014).

76. Fong, E. L. et al. Modeling Ewing sarcoma tumors in vitro with $3 \mathrm{D}$ scaffolds. Proc. Natl Acad. Sci. U.S.A. 110, 6500-6505 (2013).

77. Kim, J. W., Ho, W. J. \& Wu, B. M. The role of the 3D environment in hypoxiainduced drug and apoptosis resistance. Anticancer Res. 31, 3237-3245 (2011).

78. Huang, Y. J. \& Hsu, S. H. Acquisition of epithelial-mesenchymal transition and cancer stem-like phenotypes within chitosan-hyaluronan membrane-derived 3D tumor spheroids. Biomaterials 35, 10070-10079 (2014).

79. Tevis, K. M. et al. Mimicking the tumor microenvironment to regulate macrophage phenotype and assessing chemotherapeutic efficacy in embedded cancer cell/macrophage spheroid models. Acta Biomater. 50, 271-279 (2017).

80. Ramgolam, K. et al. Melanoma spheroids grown under neural crest cell conditions are highly plastic migratory/invasive tumor cells endowed with immunomodulator function. PLOS ONE 6, e18784 (2011).

81. Herter, S. et al. A novel three-dimensional heterotypic spheroid model for the assessment of the activity of cancer immunotherapy agents. Cancer Immunol. Immunother. 66, 129-140 (2017).

82. Phan-Lai, V. et al. CCL21 and IFN gamma recruit and activate tumor specific T cells in 3D scaffold model of breast cancer. Anticancer Agents Med. Chem. 14, 204-210 (2014).

83. Campbell, J. J. et al. Development of three-dimensional collagen scaffolds with controlled architecture for cell migration studies using breast cancer cell lines. Biomaterials 114, 34-43 (2017).

84. Provenzano, P. P. et al. Collagen density promotes mammary tumor initiation and progression. BMC Med. 6, 11 (2008).

85. Mi, K. \& Xing, Z. CD44(+)/CD24(-) breast cancer cells exhibit phenotypic reversion in three-dimensional self-assembling peptide RADA16 nanofiber scaffold. Int. J. Nanomed. 10, 3043-3053 (2015).

86. Da Sie, Y. et al. Fabrication of three-dimensional multi-protein microstructures for cell migration and adhesion enhancement. Biomed. Opt. Express 6, 480-490 (2015).

87. $\mathrm{Wu}, \mathrm{M} .$, Chen, G. \& Li, Y. P. TGF-beta and BMP signaling in osteoblast, skeletal development, and bone formation, homeostasis and disease. Bone Res. 4, 16009 (2016).

88. Taubenberger, A. V. et al. Delineating breast cancer cell interactions with engineered bone microenvironments. J. Bone Miner. Res. 28, 1399-1411 (2013).

89. Reichert, J. C. et al. Mineralized human primary osteoblast matrices as a model system to analyse interactions of prostate cancer cells with the bone microenvironment. Biomaterials 31, 7928-7936 (2010).

90. Sieh, S. et al. Interactions between human osteoblasts and prostate cancer cells in a novel 3D in vitro model. Organogenesis 6, 181-188 (2010).

91. Sieh, S. et al. Paracrine interactions between LNCaP prostate cancer cells and bioengineered bone in $3 \mathrm{D}$ in vitro culture reflect molecular changes during bone metastasis. Bone 63, 121-131 (2014).

92. Herroon, M. K., Diedrich, J. D. \& Podgorski, I. New 3D-Culture approaches to study interactions of bone marrow adipocytes with metastatic prostate cancer cells. Front. Endocrinol. 7, 84 (2016).

93. Marlow, R. et al. A novel model of dormancy for bone metastatic breast cancer cells. Cancer Res. 73, 6886-6899 (2013).

94. Fitzgerald, K. A. et al. The use of collagen-based scaffolds to simulate prostate cancer bone metastases with potential for evaluating delivery of nanoparticulate gene therapeutics. Biomaterials 66, 53-66 (2015).

95. Moreau, J. E. et al. Tissue-engineered bone serves as a target for metastasis of human breast cancer in a mouse model. Cancer Res. 67, 10304-10308 (2007).

96. Subia, B. et al. Target specific delivery of anticancer drug in silk fibroin based 3D distribution model of bone-breast cancer cells. ACS Appl. Mater. Interfaces 7, 2269-2279 (2015)

97. Cox, R. F. et al. Osteomimicry of mammary adenocarcinoma cells in vitro; increased expression of bone matrix proteins and proliferation within a 3D collagen environment. PLOS ONE 7, e41679 (2012).

98. de la Puente, $\mathrm{P}$. et al. 3D tissue-engineered bone marrow as a novel model to study pathophysiology and drug resistance in multiple myeloma. Biomaterials 73, 70-84 (2015).

99. Holzapfel, B. M. et al. Species-specific homing mechanisms of human prostate cancer metastasis in tissue engineered bone. Biomaterials 35, 4108-4115 (2014).

100. Han, Q. et al. Kinsenoside screening with a microfluidic chip attenuates gouty arthritis through inactivating NF-KB signaling in macrophages and protecting endothelial cells. Cell Death Dis. 7, e2350 (2016). 
101. Hsiao, A. Y. et al. Microfluidic system for formation of PC-3 prostate cancer coculture spheroids. Biomaterials 30, 3020-3027 (2009).

102. Buchanan, C. F. et al. Three-dimensional microfluidic collagen hydrogels for investigating flow-mediated tumor-endothelial signaling and vascular organization. Tissue Eng., Part C. 20, 64-75 (2014).

103. Ching-Te Kuo, C. - L. C. et al. Configurable 2D and 3D spheroid tissue cultures on bioengineered surfaces with acquisition of epithelial-mesenchymal transition characteristics. NPG Asia Mater. 4, e27 (2012).

104. Arai, K. et al. A novel high-throughput 3D screening system for EMT inhibitors: a pilot screening discovered the EMT inhibitory cctivity of CDK2 inhibitor SU9516. PLOS ONE 11, e0162394 (2016).

105. Mantovani, A. et al. The chemokine system in cancer biology and therapy. Cytokine Growth Factor. Rev. 21, 27-39 (2010).

106. Roussos, E. T., Condeelis, J. S. \& Patsialou, A. Chemotaxis in cancer. Nat. Rev. Cancer 11, 573-587 (2011).

107. Huang, C. P. et al. Engineering microscale cellular niches for three-dimensional multicellular co-cultures. Lab. Chip. 9, 1740-1748 (2009).

108. Vickerman, V. et al. Design, fabrication and implementation of a novel multiparameter control microfluidic platform for three-dimensional cell culture and real-time imaging. Lab. Chip. 8, 1468-1477 (2008).

109. Polacheck, W. J., Charest, J. L. \& Kamm, R. D. Interstitial flow influences direction of tumor cell migration through competing mechanisms. Proc. Natl Acad. Sci. U. S.A. 108, 11115-11120 (2011)

110. Kim, B. J. et al. Cooperative roles of SDF-1alpha and EGF gradients on tumor cell migration revealed by a robust 3D microfluidic model. PLOS ONE 8, e68422 (2013).

111. Sun, Y. S. et al. Electrotaxis of lung cancer cells in ordered three-dimensional scaffolds. Biomicrofluidics 6, 14102-1410214 (2012).

112. Shin, M. K., Kim, S. K. \& Jung, H. Integration of intra- and extravasation in one cell-based microfluidic chip for the study of cancer metastasis. Lab. Chip. 11, 3880-3887 (2011).

113. Jeon, J. S. et al. Human 3D vascularized organotypic microfluidic assays to study breast cancer cell extravasation. Proc. Natl Acad. Sci. U.S.A. 112, 214-219 (2015).

114. Bersini, S. et al. A microfluidic 3D in vitro model for specificity of breast cancer metastasis to bone. Biomaterials 35, 2454-2461 (2014).

115. Charbe, N., McCarron, P. A. \& Tambuwala, M. M. Three-dimensional bio-printing: a new frontier in oncology research. World J. Clin. Oncol. 8, 21-36 (2017).

116. $\mathrm{Ma}, \mathrm{R}$. et al. Bacterial inhibition potential of $3 \mathrm{D}$ rapid-prototyped magnesiumbased porous composite scaffolds--an in vitro efficacy study. Sci. Rep. 5, 13775 (2015).

117. Yang, Y. et al. Anti-infective efficacy, cytocompatibility and biocompatibility of a 3D-printed osteoconductive composite scaffold functionalized with quaternized chitosan. Acta Biomater. 46, 112-128 (2016).

118. Stanton, M. M., Samitier, J. \& Sanchez, S. Bioprinting of 3D hydrogels. Lab. Chip. 15, 3111-3115 (2015).

119. Vanderburgh, J., Sterling, J. A. \& Guelcher, S. A. 3D printing of tissue engineered constructs for in vitro modeling of disease progression and drug screening. Ann. Biomed. Eng. 45, 164-179 (2017).

120. Zhang, Y. S. et al. Bioprinting the cancer microenvironment. ACS Biomater. Sci. Eng. 2, 1710-1721 (2016)
121. Mou, H. et al. [Non-small cell lung cancer 95D cells co-cultured with 3Dbioprinted scaffold to construct a lung cancer model in vitro]. Zhonghua Zhongliu Zazhi 37, 736-740 (2015).

122. $\mathrm{Xu}, \mathrm{F}$. et al. A three-dimensional in vitro ovarian cancer coculture model using a high-throughput cell patterning platform. Biotechnol. J. 6, 204-212 (2011).

123. Zhao, Y. et al. Three-dimensional printing of Hela cells for cervical tumor model in vitro. Biofabrication 6, 035001 (2014).

124. Ling, K. H. G. et al. Bioprinting-based high-throughput fabrication of threedimensional MCF-7 human breast cancer cellular spheroids. Engineering 1, 269-274 (2016).

125. Grolman, J. M. et al. Rapid 3D extrusion of synthetic tumor microenvironments. Adv. Mater. 27, 5512-5517 (2015).

126. Huang, T. Q. et al. 3D printing of biomimetic microstructures for cancer cell migration. Biomed. Micro. 16, 127-132 (2014).

127. Soman, P. et al. Cancer cell migration within 3D layer-by-layer microfabricated photocrosslinked PEG scaffolds with tunable stiffness. Biomaterials 33, 7064-7070 (2012).

128. Guo, R. et al. Fabrication of 3D scaffolds with precisely controlled substrate modulus and pore size by templated-fused deposition modeling to direct osteogenic sifferentiation. Adv. Healthc. Mater. 4, 1826-1832 (2015).

129. Zhou, X. et al. 3D bioprinting a cell-laden bone matrix for breast cancer metastasis study. ACS Appl. Mater. Interfaces 8, 30017-30026 (2016).

130. Zhu, W. et al. A 3D printed nano bone matrix for characterization of breast cancer cell and osteoblast interactions. Nanotechnology 27, 315103 (2016).

131. Zhu, W. et al. 3D printed nanocomposite matrix for the study of breast cancer bone metastasis. Nanomedicine 12, 69-79 (2016).

132. Sze, W. M. R. N. \& Sai, F. From the printer: potential of three-dimensional printing for orthopaedic applications. J. Orthop. Transl. 6, 42-49 (2016).

133. Qiao, H. et al. Structural simulation of adenosine phosphate via plumbagin and zoledronic acid competitively targets JNK/Erk to synergistically attenuate osteoclastogenesis in a breast cancer model. Cell Death Dis. 7, e2094 (2016).

134. Qiao, H. et al. Synergistic suppression of human breast cancer cells by combination of plumbagin and zoledronic acid In vitro. Acta Pharmacol. Sin. 36, 1085-1098 (2015).

135. Amin, R. et al. 3D-printed microfluidic devices. Biofabrication 8, 022001 (2016).

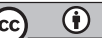

Open Access This article is licensed under a Creative Commons Attribution 4.0 International License, which permits use, sharing, adaptation, distribution and reproduction in any medium or format, as long as you give appropriate credit to the original author(s) and the source, provide a link to the Creative Commons license, and indicate if changes were made. The images or other third party material in this article are included in the article's Creative Commons license, unless indicated otherwise in a credit line to the material. If material is not included in the article's Creative Commons license and your intended use is not permitted by statutory regulation or exceeds the permitted use, you will need to obtain permission directly from the copyright holder. To view a copy of this license, visit http://creativecommons. org/licenses/by/4.0/.

(c) The Author(s) 2018 